\title{
Conhecimento dos estudantes da área da saúde acerca dos riscos dos anticoncepcionais hormonais
}

\author{
Knowledge of health care students about the risks of hormonal contraceptives \\ Conocimiento de los estudiantes de salud sobre los riesgos de los anticonceltivos \\ hormonales
}

Larissa Nobre de Lima ${ }^{1 *}$, Luiz Henrique Torres Cota ${ }^{1}$, Marina Barbosa da Costa ${ }^{1}$, Mônica Caroline de Lima Ferreira ${ }^{1}$, Paula Miranda Esteves Orsi ${ }^{1}$, Rodolfo Pereira Espíndola ${ }^{1}$, Andreia Majella da Silva Duarte Esteves ${ }^{1}$, Roberta Bessa Veloso'.

\section{RESUMO}

Objetivos: O estudo avalia o conhecimento acerca dos riscos do uso do anticoncepcional hormonal (ACO), por acadêmicas da área da saúde de uma Universidade de Minas Gerais. Métodos: Foi realizado através de questionários de uma amostra de 211 acadêmicas, no período de agosto a Outubro/2016. As variáveis consideradas foram os tipos de contraceptivos utilizados nas relações sexuais, a compreensão sobre os riscos ao usar ACO e sua respectiva recomendação. Resultados: Há um predomínio de acadêmicas menores de 25 anos e sexualmente ativas dos cursos avaliados. Sobre o conhecimento dos riscos do ACO a maioria das participantes respondeu corretamente sobre a ação do ACO ser inibida pelo uso do antibiótico, no entanto, isso não ocorreu sobre o ACO aumentar o risco de causar acidente vascular cerebral e sobre proteger contra doenças sexualmente transmissíveis. A maioria dessas acadêmicas mostrou ter conhecimento sobre o mecanismo de ação, efeito colateral, contraindicação e indicações do ACO, mas houve uma significativa parcela que não se incluiu nisso. Conclusão: Revelou-se que mesmo lidando com pessoas com maior instrução, ainda existem questões deficitárias relacionadas aos riscos do uso do ACO. Concluindo que ainda é necessária a implantação de políticas educacionais no âmbito da sexualidade.

Palavras-chaves: Anticoncepcional hormonal, Riscos, Conhecimento.

\begin{abstract}
Objectives: The study evaluates the knowledge about the risks of hormonal contraceptive use (OAC), by health academics of a University of Minas Gerais. Methods: It was conducted through questionnaires of a sample of 211 academics, from August to October / 2016. The variables considered were the types of contraceptives used in sexual intercourse, the understanding of the risks of using OAC and their recommendation. Results: There is a predominance of under- 25 and sexually active academics from the evaluated courses. About knowing the risks of OAC most participants answered correctly about the action of OAC being inhibited by antibiotic use, however, this did not occur about OAC increasing the risk of stroke and protecting against sexually transmitted diseases. Most of these academics showed to be aware of the mechanism of action, side effect, contraindication and indications of OAC, but there was a significant portion that was not included in this. Conclusion: It turned out that even dealing with people with a higher level of education, there are still deficit issues related to the risks of using OAC. Concluding that the implementation of educational policies in the scope of sexuality is still necessary.
\end{abstract}

Key words: Hormonal contraceptives, Risks, Knowledge.

1Universidade José do Rosário Vellano - Alfenas, Minas Gerais. *E-mail: larissinha_nobre@hotmail.com 


\section{RESUMEN}

Objetivos: Evalúa el conocimiento sobre riesgos del uso de anticonceptivos hormonales (OAC), por académicos de salud de una Universidad de Minas Gerais. Métodos: Realizó a través de cuestionarios de una muestra de 211 académicos, desde agosto hasta octubre/2016. Variables consideradas fueron los tipos de anticonceptivos utilizados en relaciones sexuales, comprensión de los riesgos de usar OAC y su recomendación. Resultados: Predominan académicos menores de 25 años y sexualmente activos en los cursos evaluados. Acerca de conocer los riesgos de lo OAC, la mayoría de los participantes respondieron correctamente acerca de la acción de la inhibición de lo OAC por el uso de antibióticos, sin embargo, esto no ocurrió cuando la OAC aumenta el riesgo de causar un accidente cerebrovascular y sobre la protección contra las enfermedades de transmisión sexual. Mayoría de estos académicos demostraron estar conscientes del mecanismo de acción, efectos secundarios, contraindicación y indicaciones de lo OAC, pero hubo una parte significativa que no se incluyó en esto. Conclusión: Resultó que, incluso al tratar con personas con un nivel de educación más alto, todavía hay problemas de déficit relacionados con los riesgos de usar OAC. Concluyendo que la implementación de políticas educativas en el ámbito de la sexualidad sigue siendo necesaria.

Palabras claves: Anticonceptivos hormonales, Riesgos, Conocimiento.

\section{INTRODUÇÃO}

Dentre os métodos contraceptivos, a contracepção hormonal é a mais prevalente, sendo usada por mais de 200 milhões de mulheres em todo o mundo desde a sua introdução (ARAÚJO ABR, et al., 2016). Ocorre que esse uso em excesso tem promovido riscos à saúde da população feminina mundial (OLIVEIRA MI, et al, 2016). Infelizmente, as mulheres não têm conhecimento acerca desse risco promovido pelos anticoncepcionais e isso deve ser informado pelos profissionais de saúde (BRANDT GP, et al,. 2016).

O anticoncepcional hormonal combinado oral (ACO) ou pílula anticoncepcional é um comprimido que tem em sua base a utilização de uma combinação de hormônios, geralmente estrogênio e progesterona sintéticos, com função anovulatória, além de diminuir a motilidade tubária (ARAÚJO ABR, et al, 2016). A partir disso, o progestágeno altera o muco cervical, tornando-o impenetrável para o espermatozoide e hipotrofia 0 endométrio, o qual deixa de ser capaz de implantar o embrião (WHO, 2008).

Uma das indicações para o uso da anticoncepção hormonal combinada tradicional é a sua capacidade de regular quase que cronometricamente as menstruações das pacientes, mantendo o ritmo a cada 28 dias, considerado tipicamente normal. A partir disso, com o controle do ciclo menstrual é possível evitar-se também uma anemia causada por fluxo em excesso e programar as menstruações. (RIBEIRO MTL et al, 2016).

Os anticoncepcionais orais combinados podem gerar benefícios em casos de dismenorreia, mastodinia, tensão pré-menstrual, hiperplasia e neoplasia do endométrio, endometriose, cistos funcionais e câncer de ovário, doenças benignas da mama, doenças inflamatórias pélvicas, gravidez ectópica, acne e hirsutismo. Sem dúvida alguma, a contracepção é a maior causa de prescrição de anticoncepcionais orais na prática clínica atual (ALMEIDA APF e ASSIS MM, 2017).

No entanto, vale ressaltar que há inúmeras pesquisas associando o uso prolongado de anticoncepcionais ao aparecimento de diversas condições patológicas. Entre elas destaca-se: o aumento do risco de trombose venosa e arterial, da probabilidade de acidente vascular cerebral, da predisposição ao aparecimento de carcinoma de mama, do surgimento do vírus do papiloma humano (HPV) do hipotireoidismo (PEREIRA PVS e ANGONESID 2009).

Tais condições são bem preocupantes e podem levar à morte. Cabe ressaltar aos pacientes o risco ao se tomar tal medicamento e buscar novas alternativas que não aumente o risco ao aparecimento de doenças. Além de que, o uso de anticoncepcional hormonal oral (ACO) não protege contra doenças sexualmente transmissíveis e AIDS (BRASIL, 2013). 
O trabalho é justificável, pois grande parcela da população de mulheres usa tal método contraceptivo. Ocorre que, este método é responsável por desencadear várias reações no organismo das pacientes, bem como condições patológicas na qual põe a vida da mulher em risco. Ademais, se vê um uso indiscriminado e sem orientação adequada ao uso do método. E, por isso o trabalho visa interferir por meio de palestras e cartilhas, orientando as mulheres sobre todos os métodos contraceptivos, seus respectivos mecanismos de ação, eficácia, efeitos colaterais e contraindicações. Sendo assim, a pesquisa tem como objetivo avaliar o conhecimento de acadêmicas acerca dos riscos à saúde devido ao uso dos anticoncepcionais orais, relacionando os cursos e períodos das acadêmicas.

\section{MÉTODOS}

Trata-se de uma pesquisa descritiva, do tipo transversal, com abordagem quantitativa, desenvolvida na Universidade José do Rosário Vellano. Dentre os cursos oferecidos na universidade, os de interesse para esta pesquisa foram: Biomedicina, Educação Física, Enfermagem, Farmácia, Medicina, Nutrição e Odontologia. Ademais, as alunas estavam graduando o primeiro e terceiro anos. A coleta de dados foi desenvolvida durante os meses de agosto a outubro de 2016, após a aprovação do Comitê de Ética em Pesquisa da Universidade José do Rosário Vellano (UNIFENAS) no. 1.575.716. Para tal, os pesquisadores entrevistaram todas as acadêmicas que aceitaram fazer parte da pesquisa e que assinassem o TCLE concordando em participar da mesma.

Os dados foram categorizados de acordo com as seguintes variáveis: (a) ser ou não ser sexualmente ativas; (b) a compreensão sobre os riscos ao usar anticoncepcional hormonal; (c) o conhecimento sobre as recomendações para o uso do anticoncepcional hormonal. $A$ análise dos resultados contou com a estatística descritiva e a literatura pertinente.

\section{RESULTADOS E DISCUSSÕES}

Este estudo foi realizado com 210 acadêmicas dos cursos de saúde, sendo 57 da Medicina, 31 da Odontologia, 22 da Educação Física, 21 da Farmácia, 26 da Nutrição, 31 da Biomedicina e 22 da Enfermagem. Os dados referentes à pesquisa serão descritos a seguir:

De acordo com a Tabela 1, pode-se perceber um predomínio de acadêmicas com menos de 25 anos em todos os cursos avaliados, tanto no $1^{\circ}$ quanto no $3^{\circ}$ ano. Somente no $3^{\circ}$ ano do curso de Educação Física os resultados foram iguais, ou seja, $50 \%$ da amostra referiram menos de 25 anos e $50 \%$ mais de 25 anos. A maioria das participantes referiu serem sexualmente ativas, com exceção do $1^{\circ}$ ano de medicina, onde $60 \%$ declararam não ter prática sexual, enquanto no curso de Enfermagem, também no $1^{\circ}$ ano, as médias foram iguais $(50 \%)$.

Tabela 1 - Distribuição da amostra com relação às variáveis de caracterização: faixa etária e prática sexual, entre os estudantes da área de saúde do primeiro e terceiro períodos, Alfenas-MG.

\begin{tabular}{ccccc}
\hline \multirow{2}{*}{ CURSOS E ANOS } & \multicolumn{2}{c}{ FAIXA ETÁRIA } & \multicolumn{2}{c}{ SEXUALMENTE ATIVO } \\
\cline { 2 - 5 } & $<\mathbf{2 5}$ ANOS & $>\mathbf{2 5}$ ANOS & SIM & NÃO \\
\hline Medicina 1 & $90 \%$ & $10 \%$ & $40 \%$ & $60 \%$ \\
Medicina 3 & $97 \%$ & $3 \%$ & $54 \%$ & $46 \%$ \\
Odontologia 1 & $55 \%$ & $45 \%$ & $77 \%$ & $23 \%$ \\
Odontologia 3 & $90 \%$ & $10 \%$ & $78 \%$ & $22 \%$ \\
Ed. Física 1 & $83 \%$ & $17 \%$ & $83 \%$ & $17 \%$ \\
Ed. Física 3 & $50 \%$ & $50 \%$ & $70 \%$ & $30 \%$ \\
Farmácia 1 & $88 \%$ & $12 \%$ & $100 \%$ & $0 \%$ \\
Farmácia 3 & $66 \%$ & $34 \%$ & $58 \%$ & $42 \%$ \\
Nutrição 1 & $88 \%$ & $12 \%$ & $53 \%$ & $47 \%$ \\
Nutrição 3 & $77 \%$ & $23 \%$ & $67 \%$ & $33 \%$ \\
Biomedicina 1 & $90 \%$ & $10 \%$ & $60 \%$ & $40 \%$ \\
Biomedicina 3 & $66 \%$ & $34 \%$ & $72 \%$ & $28 \%$ \\
Enfermagem 1 & $87 \%$ & $13 \%$ & $50 \%$ & $50 \%$ \\
\hline
\end{tabular}

Fonte: Lima LN, et al., 2018. Siglas: ed. física: educação física. 
De acordo com Brito MB et al (2011), atualmente, percebe-se muitos jovens que iniciam as atividades sexuais cada vez mais cedo, mesmo não sendo admitido para muitos pais esse tipo de comportamento. $E$ como consequência, têm ocorrido muitos problemas tanto no colégio quanto na sociedade e no projeto de vida desses jovens, que por muitas vezes acabam engravidando ou contraindo uma Doença Sexualmente Transmissível (DST), ocasionando problemas graves no futuro.

Diante desta realidade, e para que ocorra uma iniciação sexual segura, é fundamental que os adolescentes tenham acesso a métodos contraceptivos e a informações referentes aos riscos advindos de relações sexuais desprotegidas (SASAKI RSA, 2015).

Entre os fatores que estão associados ao início precoce da vida sexual, pode-se citar a baixa inserção socioeconômica, a cor da pele não branca, o frágil vínculo familiar e escolar e o uso de drogas lícitas e ilícitas (MADKOUR AS, et al., 2010).

Assim, já na universidade, os jovens dão continuidade à sua vida sexual mesmo que de maneira errônea, sem uso de preservativos, ou nenhum modo de proteção. Muitos precisam se ausentar da faculdade devido à gravidez, atrasando os estudos e tendo que repor aulas posteriormente.

Pela Tabela 2, analisando-se as acadêmicas do $1^{\circ}$ e $3^{\circ}$ anos dos referidos cursos pode-se observar a referência à ação inibitória do anticoncepcional hormonal oral (ACO) com o uso de antibiótico (ATB) ela maioria delas, com exceção das participantes do $1^{\circ}$ ano de Biomedicina e Nutrição onde a maioria $(60 \%$ e $52 \%$, respectivamente) referiu não saber. Nenhuma das participantes assinalou que o uso de antibióticos ajuda na ação dos ACO.

Anticoncepcionais orais são formados pela associação de estrogênio e a progesterona, em doses reduzidas, como mecanismo de ação inibindo a ovulação, modificando a superfície uterina e o muco cervical, além de promover dificuldade da passagem dos espermatozoides.

Ademais, o uso concomitante de determinados antibióticos, faz com que as doses hormonais diminuem, comprometendo sua eficácia. Outro ponto importante é que tais medicamentos destroem as bactérias intestinais que produzem as enzimas que podem reagir com o estrogênio para sua absorção pela corrente sanguínea e provocar a aceleração do metabolismo hepático que compromete a absorção dos outros hormônios, prejudicando assim a ação contraceptiva. Exemplos como a ampicilina e doxiciclina, podem causar adversidades se empregados em conjunto com a pílula anticoncepcional (MATTOS JM, 2012).

Se tomada juntamente com antibióticos, o anticoncepcional pode não fazer efeito, tornando a mulher desprotegida contra uma gravidez não planejada. Em 1971, foi feito o primeiro estudo em relação a ação dos antibióticos em anticoncepcionais e desde então, têm sido estudados por diversos pesquisadores (PAIVA V, et al, 2008).

De acordo com o uso de o ACO aumentar o risco de ter AVC (Tabela 2), nos primeiros anos do curso foi citado pela maioria das participantes dos cursos de Medicina $80 \%(n=16)$ e Odontologia em 55,6\% $(n=5)$, enquanto no $3^{\circ}$ ano, pela Medicina 78,3\% ( $\left.n=29\right)$, Farmácia $58,1 \%(n=7)$ e Enfermagem $57,1 \%(n=8)$. Já os que discordam e referiram que o uso do ACO diminui o risco de ter AVC foi referido por $11,1 \%(n=1)$ das participantes do curso de Odontologia do $1^{\circ}$ ano e $8,7 \%(n=1)$ do curso de Farmácia do $3^{\circ}$.

Entretanto um dado preocupante, em se tratando de acadêmicas da área de saúde, é o fato de que a maioria das entrevistadas referiu não saber, com destaque para os primeiros anos de $81,7 \%(n=9)$ da Educação física, 92,8\% $(n=15)$ da Nutrição e $85 \%(n=13)$ da Biomedicina e dos terceiros anos $81,7 \%(n=18)$ da Odontologia, 77,8\% ( $n=7)$ da Nutrição e $81 \%(n=10)$ da Biomedicina.

$A$ associação do AVC isquêmico com o uso de ACO foi bem avaliada em muitos estudos. Foi visto em muitos resultados em que havia uma maior prevalência de AVC em pacientes usuárias de anticoncepcionais, além de que foi observada uma relativa proteção em mulheres que interromperam o uso dessa droga. Porém, todos estes estudos são apenas observacionais, pois para se fazer um estudo de caso-controle, o grupo que estivesse recebendo o placebo, não teria a anticoncepção e isso seria antiético (CARLINI BC, et al., 2011). 
Ainda de acordo com a Tabela 2, quando questionadas sobre a relação da proteção contra as DSTs e o uso do ACO, evidenciou-se pela maioria de todos os cursos um desconhecimento sobre o assunto, ou seja, as entrevistadas não souberam responder. Outro fato alarmante é que algumas ainda citaram que o uso do ACO protege a mulher contra as DSTs, com destaque para o $3^{\circ}$ ano do curso de Medicina, onde $35,1 \%(\mathrm{~N}=13)$ das entrevistadas referiram tal proteção.

Tabela 2 - Distribuição da amostra com relação ao conhecimento acerca dos riscos dos anticoncepcionais hormonais de acordo com os estudantes da área de saúde do primeiro e terceiro períodos, Alfenas-MG.

\begin{tabular}{lccccccccc}
\hline & \multicolumn{3}{c}{$\begin{array}{c}\text { Ação do aco com o uso } \\
\text { do ATB }\end{array}$} & \multicolumn{2}{c}{ Risco de ter AVC } & \multicolumn{3}{c}{ infecção por DSTS } \\
\cline { 2 - 10 } Cursos e anos & Inibe & Ajuda & $\begin{array}{c}\text { Não } \\
\text { sabe }\end{array}$ & Aumenta & Diminui & $\begin{array}{c}\text { Não } \\
\text { sabe }\end{array}$ & $\begin{array}{c}\text { Risco de } \\
\text { contrair }\end{array}$ & Proteger & $\begin{array}{c}\text { Não } \\
\text { sabe }\end{array}$ \\
\hline Medicina 1 & $85,0 \%$ & $0,0 \%$ & $15,0 \%$ & $80,0 \%$ & $0,0 \%$ & $20,0 \%$ & $20,0 \%$ & $2,5 \%$ & $77,5 \%$ \\
Medicina 3 & $100,0 \%$ & $0,0 \%$ & $0,0 \%$ & $78,3 \%$ & $0,0 \%$ & $21,7 \%$ & $24,3 \%$ & $35,1 \%$ & $40,6 \%$ \\
Odontologia 1 & $100,0 \%$ & $0,0 \%$ & $0,0 \%$ & $55,6 \%$ & $11,1 \%$ & $33,3 \%$ & $16,6 \%$ & $16,7 \%$ & $66,7 \%$ \\
Odontologia 3 & $86,3 \%$ & $0,0 \%$ & $13,7 \%$ & $18,3 \%$ & $0,0 \%$ & $81,7 \%$ & $6,9 \%$ & $11,4 \%$ & $81,7 \%$ \\
Ed. Física 1 & $55,6 \%$ & $0,0 \%$ & $44,4 \%$ & $18,3 \%$ & $0,0 \%$ & $81,7 \%$ & $21,0 \%$ & $9,1 \%$ & $69,9 \%$ \\
Ed. Física 3 & $74,7 \%$ & $0,0 \%$ & $25,3 \%$ & $33,6 \%$ & $0,0 \%$ & $66,4 \%$ & $17,0 \%$ & $0,0 \%$ & $83,0 \%$ \\
Farmácia 1 & $88,9 \%$ & $0,0 \%$ & $11,1 \%$ & $44,4 \%$ & $0,0 \%$ & $55,6 \%$ & $16,7 \%$ & $11,1 \%$ & $72,2 \%$ \\
Farmácia 3 & $91,3 \%$ & $0,0 \%$ & $8,7 \%$ & $59,1 \%$ & $8,7 \%$ & $32,2 \%$ & $33,6 \%$ & $0,0 \%$ & $66,4 \%$ \\
Nutrição 1 & $48,0 \%$ & $0,0 \%$ & $52,0 \%$ & $7,2 \%$ & $0,0 \%$ & $92,8 \%$ & $18,4 \%$ & $3,3 \%$ & $78,3 \%$ \\
Nutrição 3 & $78,0 \%$ & $0,0 \%$ & $22,0 \%$ & $22,2 \%$ & $0,0 \%$ & $77,8 \%$ & $11,1 \%$ & $16,7 \%$ & $72,2 \%$ \\
Biomedicina 1 & $40,0 \%$ & $0,0 \%$ & $60,0 \%$ & $15,0 \%$ & $0,0 \%$ & $85,0 \%$ & $9,5 \%$ & $8,0 \%$ & $82,5 \%$ \\
Biomedicina 3 & $54,0 \%$ & $0,0 \%$ & $46,0 \%$ & $19,0 \%$ & $0,0 \%$ & $81,0 \%$ & $14,5 \%$ & $0,0 \%$ & $85,5 \%$ \\
Enfermagem 1 & $62,5 \%$ & $0,0 \%$ & $37,5 \%$ & $37,5 \%$ & $0,0 \%$ & $62,5 \%$ & $0,0 \%$ & $25,0 \%$ & $75,0 \%$ \\
Enfermagem 3 & $57,1 \%$ & $0,0 \%$ & $42,9 \%$ & $57,1 \%$ & $0,0 \%$ & $42,9 \%$ & $10,7 \%$ & $7,2 \%$ & $82,1 \%$ \\
\hline
\end{tabular}

Fonte: Lima LN, et al., 2018. Siglas: ACO: anticoncepcional hormonal oral; AVC: acidente vascular cerebral; DST: doenças sexualmente transmissíveis.

Como se sabe, os anticoncepcionais não evitam a transmissão de DST's entre os parceiros, eles fazem um controle hormonal que apenas evitam uma eventual gestação indesejada pelo casal. Por isso, mesmo se a mulher estiver fazendo uso de pílulas anticoncepcionais, é recomendado o uso de preservativo em todas as relações sexuais, pois não estão imunes a transmissão das DST's, por esse contágio ser feito pelo contato com os fluídos. (CUNHA GO e GALVÃO MTG, 2011).

Ainda de acordo com as autoras os anticoncepcionais são medicamentos que, quando usados corretamente, previnem a gravidez. São eficientes para este meio e costumam funcionar bem se tomados conforme indica a bula, com as devidas pausas e mantendo-se o horário da ingestão.

Segundo os dados obtidos na Tabela 3, os principais mecanismos de ação do ACO obtidos na pesquisa foram sobre a inibição da ovulação e controle do fluxo menstrual. Entretanto, também foram citados, embora que pela minoria, matar os espermatozoides, diminuição da motilidade tubária e proteção de DSTs. Com relação à contraindicação para o uso dos $A C O$ destacam-se no $1^{\circ}$ ano que 5 cursos referiram em sua maioria não saber se há contraindicação para a prescrição, sendo Educação física, Farmácia, Nutrição, Biomedicina e Enfermagem.

Já no $3^{\circ}$ ano, 4 cursos referiram desconhecer as contraindicações, sendo Odontologia, Educação física, Nutrição e Biomedicina. Ao serem questionadas se o ACO pode ocasionar efeitos colaterais, a maioria dos cursos, tanto do $1^{\circ}$ quanto do $3^{\circ}$ ano, referiram que apresentam, entretanto os cursos de Farmácia e Biomedicina do $1^{\circ}$ ano e Odontologia e Nutrição do $3^{\circ}$ referiram não saber responder. Acerca das principais indicações do ACO as mais citadas foram: evitar gravidez, disfunção menstrual, síndrome do ovário policístico e controle do ciclo menstrual. 
Tabela 3 - Distribuição da amostra com relação às variáveis: mecanismos de ação, contraindicação, efeito colateral dos anticoncepcionais hormonais de acordo com os estudantes da área de saúde do primeiro e terceiro períodos, Alfenas-MG.

\begin{tabular}{|c|c|c|c|c|c|c|c|c|c|c|c|}
\hline \multirow[b]{2}{*}{$\begin{array}{c}\text { Cursos e } \\
\text { anos }\end{array}$} & \multicolumn{7}{|c|}{ Mecanismo de ação } & \multicolumn{2}{|c|}{$\mathrm{Cl}$} & \multicolumn{2}{|c|}{ EC } \\
\hline & $\begin{array}{c}\text { Inibe a } \\
\text { ovulaçã } \\
0\end{array}$ & $\begin{array}{l}\text { Altera o } \\
\text { muco } \\
\text { cervical }\end{array}$ & $\begin{array}{l}\text { Mat } \\
\text { a os } \\
\text { epz. }\end{array}$ & $\begin{array}{l}\text { Diminui } \\
\text { motilidad } \\
\text { e tubária }\end{array}$ & $\begin{array}{c}\text { Controla } \\
\text { o fluxo } \\
\text { menstru } \\
\text { al }\end{array}$ & $\begin{array}{l}\text { Proteg } \\
\text { e } \\
\text { contra } \\
\text { DST }\end{array}$ & $\begin{array}{c}\text { Altera } \\
\text { endométri } \\
0\end{array}$ & Sim & Não & Sim & Não \\
\hline Medicina 1 & $90 \%$ & $10 \%$ & $0 \%$ & $5 \%$ & $35 \%$ & $0 \%$ & $0 \%$ & $65 \%$ & $35 \%$ & $75 \%$ & $25 \%$ \\
\hline Medicina 3 & $84 \%$ & $21 \%$ & $0 \%$ & $0 \%$ & $81 \%$ & $2 \%$ & $13 \%$ & $75 \%$ & $25 \%$ & $95 \%$ & $5 \%$ \\
\hline Odontologia 1 & $100 \%$ & $0 \%$ & $0 \%$ & $0 \%$ & $88 \%$ & $0 \%$ & $0 \%$ & $78 \%$ & $22 \%$ & $100 \%$ & $0 \%$ \\
\hline Odontologia 3 & $95 \%$ & $0 \%$ & $0 \%$ & $0 \%$ & $36 \%$ & $9 \%$ & $0 \%$ & $40 \%$ & $60 \%$ & $45 \%$ & $55 \%$ \\
\hline Ed. Física 1 & $42 \%$ & $0 \%$ & $16 \%$ & $0 \%$ & $83 \%$ & $0 \%$ & $8 \%$ & $0 \%$ & $100 \%$ & $67 \%$ & $33 \%$ \\
\hline Ed. Física 3 & $40 \%$ & $0 \%$ & $0 \%$ & $0 \%$ & $50 \%$ & $0 \%$ & $10 \%$ & $30 \%$ & $70 \%$ & $50 \%$ & $50 \%$ \\
\hline Farmácia 1 & $100 \%$ & $10 \%$ & $0 \%$ & $0 \%$ & $44 \%$ & $0 \%$ & $0 \%$ & $33 \%$ & $67 \%$ & $44 \%$ & $56 \%$ \\
\hline Farmácia 3 & $91 \%$ & $0 \%$ & $0 \%$ & $0 \%$ & $50 \%$ & $0 \%$ & $0 \%$ & $58 \%$ & $42 \%$ & $83 \%$ & $17 \%$ \\
\hline Nutrição 1 & $41 \%$ & $0 \%$ & $11 \%$ & $0 \%$ & $53 \%$ & $12 \%$ & $0 \%$ & $41 \%$ & $59 \%$ & $64 \%$ & $36 \%$ \\
\hline Nutrição 3 & $55 \%$ & $0 \%$ & $11 \%$ & $0 \%$ & $33 \%$ & $22 \%$ & $0 \%$ & $45 \%$ & $55 \%$ & $33 \%$ & $67 \%$ \\
\hline Biomedicina 1 & $40 \%$ & $0 \%$ & $20 \%$ & $0 \%$ & $45 \%$ & $0 \%$ & $0 \%$ & $20 \%$ & $80 \%$ & $37 \%$ & $63 \%$ \\
\hline Biomedicina 3 & $63 \%$ & $27 \%$ & $0 \%$ & $18 \%$ & $45 \%$ & $0 \%$ & $18 \%$ & $36 \%$ & $64 \%$ & $54 \%$ & $46 \%$ \\
\hline Enfermagem 1 & $37 \%$ & $0 \%$ & $12 \%$ & $0 \%$ & $62 \%$ & $0 \%$ & $0 \%$ & $38 \%$ & $62 \%$ & $62 \%$ & $38 \%$ \\
\hline Enfermagem 3 & $93 \%$ & $21 \%$ & $14 \%$ & $7 \%$ & $36 \%$ & $7 \%$ & $0 \%$ & $57 \%$ & $43 \%$ & $57 \%$ & $43 \%$ \\
\hline
\end{tabular}

Fonte: Lima LN, et al., 2018. Siglas: epz: espermatozoide; DST: doença sexualmente transmissível; Cl: contra-indicação; EC: efeito colateral.

Na Tabela 4, são indicados os resultados da pesquisa sobre o conhecimento das estudantes acerca das indicações dos ACOs. Dentre as indicações, foi questionado sobre evitar a gravidez e tratamento para disfunção menstrual, Tensão pré-menstrual (TPM), hemorragia disfuncional, Síndrome dos ovários polucísticos, controle do ciclo menstrual e acne.

Tabela 4 - Distribuição da amostra com relação sobre o conhecimento das indicações dos anticoncepcionais hormonais de acordo com os estudantes da área de saúde do primeiro e terceiro períodos, Alfenas-MG.

\begin{tabular}{|c|c|c|c|c|c|c|c|}
\hline \multirow[b]{2}{*}{ Cursos e anos } & \multicolumn{7}{|c|}{ Indicações } \\
\hline & $\begin{array}{c}\text { Evitar } \\
\text { gravidez }\end{array}$ & $\begin{array}{l}\text { Disfunção } \\
\text { menstrual }\end{array}$ & TPM & $\begin{array}{l}\text { Hemorragia } \\
\text { disfuncional }\end{array}$ & $\begin{array}{l}\text { Síndrome } \\
\text { ovário } \\
\text { policístico }\end{array}$ & $\begin{array}{l}\text { Controle do } \\
\text { ciclo } \\
\text { menstrual }\end{array}$ & Acne \\
\hline Medicina 1 & $100 \%$ & $90 \%$ & $65 \%$ & $15 \%$ & $80 \%$ & $80 \%$ & $65 \%$ \\
\hline Medicina 3 & $100 \%$ & $83 \%$ & $51 \%$ & $13 \%$ & $64 \%$ & $78 \%$ & $43 \%$ \\
\hline Odontologia 1 & $88 \%$ & $55 \%$ & $44 \%$ & $11 \%$ & $66 \%$ & $88 \%$ & $88 \%$ \\
\hline Odontologia 3 & $95 \%$ & $36 \%$ & $22 \%$ & $10 \%$ & $27 \%$ & $54 \%$ & $50 \%$ \\
\hline Ed. Física 1 & $91 \%$ & $42 \%$ & $17 \%$ & $17 \%$ & $34 \%$ & $68 \%$ & $25 \%$ \\
\hline Ed. Física 3 & $70 \%$ & $20 \%$ & $40 \%$ & $10 \%$ & $20 \%$ & $40 \%$ & $20 \%$ \\
\hline Farmácia 1 & $88 \%$ & $44 \%$ & $22 \%$ & $1 \%$ & $22 \%$ & $33 \%$ & $44 \%$ \\
\hline Farmácia 3 & $83 \%$ & $75 \%$ & $41 \%$ & $17 \%$ & $58 \%$ & $50 \%$ & $58 \%$ \\
\hline Nutrição 1 & $82 \%$ & $29 \%$ & $23 \%$ & $6 \%$ & $35 \%$ & $65 \%$ & $23 \%$ \\
\hline Nutrição 3 & $88 \%$ & $55 \%$ & $55 \%$ & $22 \%$ & $22 \%$ & $55 \%$ & $44 \%$ \\
\hline Biomedicina 1 & $75 \%$ & $15 \%$ & $20 \%$ & $10 \%$ & $20 \%$ & $10 \%$ & $30 \%$ \\
\hline Biomedicina 3 & $90 \%$ & $27 \%$ & $63 \%$ & $9 \%$ & $45 \%$ & $27 \%$ & $27 \%$ \\
\hline Enfermagem 1 & $62 \%$ & $25 \%$ & $0 \%$ & $25 \%$ & $25 \%$ & $50 \%$ & $37 \%$ \\
\hline Enfermagem 3 & $100 \%$ & $43 \%$ & $28 \%$ & $21 \%$ & $14 \%$ & $43 \%$ & $7 \%$ \\
\hline
\end{tabular}

Fonte: Lima LN, et al., 2018. Siglas: TPM: tensão pré-menstrual. 
Os anticoncepcionais ou contraceptivos orais são fármacos que previnem a gravidez e podem ser utilizados em circunstâncias específicas como na prevenção de uma gravidez de risco, no planejamento familiar, controle do crescimento populacional, entre outras. Apresentam também outros benefícios tais como: a regularização do ciclo menstrual, redução da tensão pré-menstrual, redução da incidência de cistos ovarianos, de câncer ovariano e endometrial e de doenças benignas das mamas (LUBIANCA JN e WANNMACHER L, 2011).

Ou seja, os contraceptivos orais combinados funcionam através de três principais mecanismos de ação. Após serem ingeridos, são absorvidos no intestino e passam à corrente sanguínea. Através do sangue, circulam e chegam à hipófise e aos ovários, impedindo a ovulação. Também fazem com que o muco do colo uterino (muco cervical) se torne mais espesso, de forma a impedir a passagem dos espermatozoides. $O$ terceiro mecanismo de ação consiste em evitar que o endométrio esteja adequadamente preparado para a gravidez (HEINEMANN LA e LUBIANCA JN, 2010). Em relação à contraindicação, o ideal é que a pílula seja usada somente após uma consulta detalhada, onde serão investigadas possíveis contraindicações para o uso do contraceptivo hormonal. Como todas as drogas, os anticoncepcionais são contraindicados em algumas situações e sofrem interações quando tomados com outros medicamentos, como alguns antibióticos, que podem diminuir o efeito da pílula e aumentar o risco de gestação indesejada (VAN VLIET H, et al, 2010).

Sobre os efeitos colaterais, os mais indesejáveis relacionados ao uso prolongado dos contraceptivos hormonais é o aumento de diversos riscos para a saúde, como o aumento de duas a três vezes a chance de desenvolver eventos trombóticos, como a trombose venosa profunda, o acidente vascular cerebral e o infarto agudo do miocárdio. No entanto, este risco diminui com o maior tempo de uso e em relação à menor dose de estrogênio. Também sendo visto que este risco diminui após a descontinuação de seu uso. (WONG CL, et al., 2010). Por fim, é um método indicado para pessoas que não querem ou não podem engravidar no momento, que possuem algum problema no ovário ou até mesmo para o tratamento de alguma patologia específica (CIBULA D, et al, 2010).

\section{CONCLUSÃO}

Esse estudo revelou que mesmo lidando com pessoas com um maior grau de instrução, ainda existem questões deficitárias que envolvem os riscos do uso do anticoncepcional hormonal. Os resultados encontrados mostram a maioria das acadêmicas dos referidos cursos referiu que a ação do ACO usado juntamente com ATB é inibida, com exceção das participantes do $1^{\circ}$ ano de Biomedicina onde a maioria (60\%) referiu não saber. Realmente os antibióticos diminuem a eficácia do anticoncepcional hormonal, porém, esperava-se uma maior assertividade na resposta, principalmente vindo de estudantes do ensino superior $\mathrm{e}$ da área da saúde. Em relação ao uso de o ACO aumentar o risco de ter AVC, a maioria das acadêmicas concordou com essa afirmação. Entretanto, no geral, várias acadêmicas disseram não saber sobre essa influência, fato este que preocupa, já que esse tema é assunto básico referido nos referentes cursos. Outro dado preocupante foi o fato da maioria das estudantes referir não saber sobre a relação do uso do ACO e da proteção contra a IST, algumas ainda citaram que o uso do ACO protege a mulher contra as ISTs, com destaque para o $3^{\circ}$ ano do curso de Medicina, o que não procede. Com base nos dados pode-se concluir que, mesmo lidando com pessoas de um alto nível intelectual, ainda se faz necessária a implantação de políticas educacionais ou campanhas no âmbito da sexualidade, visando à orientação de jovens quanto ao uso e riscos que o ACO pode trazer a saúde da mulher.

\section{REFERÊNCIAS}

1. ALMEIDA APF, ASSIS MM. Efeitos colaterais e alterações fisiológicas relacionadas ao uso contínuo de anticoncepcionais hormonais orais. Revista Eletrôn. Atualiza Saúde, Salvador. 2017. v. 5, n. 5, p. 85-93.

2. ARAÚJO ABR, et al. Anticoncepcionais hormonais contendo apenas progestágenos e seus principais efeitos. Brazilian Journal of Surgery and Clinical Research. 2016. V.15, n.1, p.75-81.

3. BRANDT GP, RODRIGUES AP, BURCI LM. Conhecimento de usuárias de anticoncepcionais orais acerca de hábitos e interações medicamentosas em uma unidade básica de saúde. Visão Acadêmica, Curitiba. 2016. v.17 n.4. 
4. BRASIL, MINISTÉRIO DA SAÚDE. Saúde sexual e saúde reprodutiva. Brasília: Ministério da Saúde. 2013. p.126.

5. BRITO MB, NOBRE F, VIEIRA CS. Contracepção Hormonal e Sistema Cardiovascular Hormonal. Faculdade de Medicina de Ribeirão Preto - Universidade de São Paulo, São Paulo, SP - Brasil, 2011.

6. CARLINI BC, GAZAL CC, GOUVEIA N. Comportamento de saúde entre jovens estudantes das redes pública e privada da área metropolitana de São Paulo. Revista de Saúde Pública. 2011. 34, 636-45.

7. CIBULA D, et al. L. Hormonal contraception and risk of cancer. Hum. Reprod. Update, Oxford. 2010. v. 16, n. 6, p. 631-650.

8. CUNHA GO, GALVÃO MTG. Métodos contraceptivos e de prevenção da transmissão/reinfecção do vírus entre portadores de hiv/aids. Universidade Federal do Ceará (UFC), 2011.

9. DEVELOPMENT CORE TEAM (2016). R: A language and environment for statistical computing. R Foundation for Statistical Computing, Vienna, Austria. ISBN 3-900051-07-0, URL http://www.R-project.org. Acesso em novembro de 2017.

10. HEINEMANN LA, LUBIANCA JN. Use of oral contraceptives containing gestodene and risk of venous thromboembolism: outlook 10 years after the third-generation "pill scare". Contraception, Stoneham. 2010. v. 81, n. 5, p. 401-407.

11. LUBIANCA JN, WANNMACHER L. Uso Racional de Contraceptivos Hormonais Orais. Comissão Nacional Especializada de Anticoncepção da Federação Brasileira de Ginecologia e Obstetrícia FEBRASGO, 2011.

12. MADKOUR AS, et al. Early Adolescent Sexual Initiation as a Problem Behavior: A Comparative Study of Five Nations. Journal of Adolescent Health. 2010. 47, 389-398.

13. MATTOS JM. Pílulas anticoncepcionais. Universidade Estadual de Campinas. PIBID-IQ-UNICAMP, 2012.

14. OLIVEIRA MI, et al. Investigação sobre uso de anticoncepcionais hormonais: ciência dos riscos para a saúde. Anais do 8을ão Internacional de Ensino, Pesquisa e Extensão - Universidade Federal do Pampa. 2016. V. 8, n. 1.

15. PAIVA V, et al. Idade e uso de preventivo na iniciação sexual de adolescentes brasileiros. Revista de Saúde Pública, 2008. v. 42, 45-53.

16. PEREIRA PVS, ANGONESI D. Efeito do uso prolongado de contraceptivos orais. Infarma. Brasília. 2009. v. 21, n. $7 / 8$, p. $21-28$.

17. RIBEIRO MTL, et al. Avaliação do uso de hormônios anticoncepcionais e reposição hormonal por alunas de uma Instituição de Ensino Superior. Revista de Ciências Farmacêutica Básica e Aplicada, Araraquara. 2016. v. 37 Supl. 1.

18. SASAKI RSA. Prevalência de relação sexual e fatores associados em adolescentes escolares de Goiânia, Goiás, Brasil. Ciência e Saúde Coletiva. 2015. 20(1), 95-104.

19. VAN VLIET H, et al. Biphasic versus monophasic oral contraceptives for contraception. Cochrane Database of Systematic Reviews, Oxford. 2010. Issue 10.

20. WHO, World Health Organization. Reproductive Health and Research and John Hopkins Bloomberg School of Public Health. Family planning: a global handbook for providers. Baltimore and Geneva: CCP and WHO, 2008.

21. WONG CL, et al. Oral contraceptive pill for primary dysmenorrhoea. Cochrane Database of Systematic Reviews. Oxford. 2010. Issue 10. 